\title{
LATTICES WITHOUT SHORT CHARACTERISTIC VECTORS
}

\author{
Mark Gaulter
}

\begin{abstract}
All the lattices here under discussion here are understood to be integral unimodular $\mathbb{Z}$-lattices in $\mathbb{R}^{n}$. A characteristic vector of a lattice $L$ is a vector $w \in L$ such that $v \cdot w \equiv|v|^{2}(\bmod 2)$ for every $v \in L$. Elkies has considered the minimal (squared) norm of the characteristic vectors in a unimodular lattice. He showed that any unimodular $\mathbb{Z}$-lattice in $\mathbb{R}^{n}$ has characteristic vectors of norm $\leq n$; he also proved that of all such lattices, only the standard lattice $\mathbb{Z}^{n}$ has no characteristic vectors of norm $<n$ (Math Research Letters 2, 321-326). He then asked "For any $k>0$, is there $\mathcal{N}_{k}$ such that every integral unimodular lattice all of whose characteristic vectors have norm $\geq n-8 k$ is of the form $L_{0} \perp \mathbb{Z}^{r}$ for some lattice $L_{0}$ of rank at most $\mathcal{N}_{k}$ ?" (Math Research Letters 2, 643-651). He solved this question in the case $k=1$, showing that $\mathcal{N}_{1}=23$ suffices; here I determine values for $\mathcal{N}_{2}$ and $\mathcal{N}_{3}$.
\end{abstract}

\section{Introduction}

A $\mathbb{Z}$-lattice is a free module of finite rank over $\mathbb{Z}$. Given a $\mathbb{Z}$-lattice $L$, let $B: L \times L \rightarrow \mathbb{Z}$ be a symmetric bilinear form and $q: L \rightarrow \mathbb{Z}$ given by $q(x)=B(x, x)$ the corresponding quadratic form. Throughout this paper we will assume that $q$ is positive definite. This enables us to embed $L$ in $\mathbb{R}^{n}$, with $B(\cdot, \cdot)$ the standard inner product and $q(\cdot)$ the corresponding (squared) norm. A characteristic vector of $L$ is an element $w$ such that $B(v, w) \equiv q(v)(\bmod 2)$ for every $v \in L$. Characteristic vectors are known to exist in any unimodular $\mathbb{Z}$-lattice $L$, and in this case they constitute a coset of $2 L$ in $L$. If $L$ has rank $n$, all the characteristic elements have norm congruent to $n(\bmod 8)($ see $[\mathrm{B}]$; or see Chapter V of $[\mathrm{S}])$.

Noam Elkies has considered the minimal norm of the characteristic vectors in a unimodular lattice. In [E1], Elkies shows that any positive definite unimodular $\mathbb{Z}$-lattice of rank $n$ has characteristic vectors of norm $\leq n$; he also proves that of all such lattices, only the standard lattice $\mathbb{Z}^{n}$ has no characteristic vectors of norm strictly less than $n$. Then in [E2], he begins a programme of showing that a positive definite unimodular lattice whose minimal characteristic vectors have norm close to $n$ are in some sense close to $\mathbb{Z}^{n}$. More precisely, he shows that every such lattice whose characteristic vectors all have norm $\geq n-8$ is of the form $L_{0} \perp \mathbb{Z}^{r}$ for some $L_{0}$ of rank $\leq 23$. He then asks: "For any $k>0$, is there $\mathcal{N}_{k}$ such that every integral [positive definite] unimodular lattice all of whose characteristic vectors have norm $\geq n-8 k$ is of the form $L_{0} \perp \mathbb{Z}^{r}$ for

Received January 15, 1998. 
some lattice $L_{0}$ of rank at most $\mathcal{N}_{k}$ ?" Elkies goes on to comment: "Even the case $k=2$ appears difficult."

In this paper, we first obtain upper bounds on the number of characteristic vectors of minimal norm $s$ and on the number of characteristic vectors of norm $s+8$; then we apply a theorem of Hecke to settle the cases $k=2$ and $k=3$ of Elkies' problem.

\section{Notation}

We will largely follow the notation of [O'M]. Also, for a given lattice $L$, we define:

$$
\begin{aligned}
\chi=\chi_{L} & :=\{v \in L: B(x, v) \equiv q(x)(\bmod 2), \forall x \in L\} \\
\chi_{t}=\chi_{t}(L) & :=\left\{v \in \chi_{L}: q(v)=t\right\} \\
s=s(L) & :=\min _{v \in \chi_{L}}\{q(v)\} .
\end{aligned}
$$

Thus $\chi_{s}$ denotes the set of shortest characteristic vectors of the lattice $L$ under discussion. Finally, for any set $\mathcal{A}$, define $|\mathcal{A}|$ to be the cardinality of $\mathcal{A}$.

\section{A bound on the number of shortest characteristic vectors}

Throughout this section, $L$ denotes a positive definite unimodular $\mathbb{Z}$-lattice of rank $n$. We will find bounds on $\left|\chi_{s}\right|$ and $\left|\chi_{s+8}\right|$. The characteristic elements of $L$ constitute a coset of $2 L$ in $L$, so if $v_{1}, v_{2} \in \chi_{L}$ then $v_{1}+v_{2} \in 2 L$. If $v_{1}, v_{2}$ have the same norm, we can say more:

Lemma 3.1. Let $v_{1}, v_{2}$ be characteristic elements of $L$ with $q\left(v_{1}\right)=q\left(v_{2}\right)=t$. Then

$$
q\left(\frac{v_{1}+v_{2}}{2}\right) \leq t
$$

with equality if and only if $v_{1}=v_{2}$.

Proof. This is because a ball in Euclidean space is strictly convex.

Lemma 3.2. Fix $w \in \chi_{s}$. Define the map $\phi_{w}: \chi_{s} \rightarrow L / 2 L$ by

$$
\phi_{w}(v):=\frac{v-w}{2}+2 L .
$$

Then $\phi_{w}$ is injective.

Proof. Suppose $\phi_{w}\left(v_{1}\right)=\phi_{w}\left(v_{2}\right)$. Then $\frac{v_{1}-v_{2}}{2} \in 2 L$, from which we see

$$
\frac{v_{1}+v_{2}}{2}=v_{2}+\frac{v_{1}-v_{2}}{2} \in \chi_{L}
$$

Therefore

$$
q\left(\frac{v_{1}+v_{2}}{2}\right) \geq s
$$

But $v_{1}, v_{2} \in \chi_{s}$, so by Lemma 3.1 we have $q\left(\frac{v_{1}+v_{2}}{2}\right) \leq s$. Thus we have equality, and by applying Lemma 3.1 again we see $v_{1}=v_{2}$, as required. 
Lemma 3.2 gives us an injective function from $\chi_{s}$ into a group of order $2^{n}$. This proves the following:

Corollary 3.3. The number of shortest characteristic vectors of a positive definite unimodular $\mathbb{Z}$-lattice of dimension $n$ is at most $2^{n}$.

This result is the best possible, as the following example shows. Let $\left\{e_{1}, e_{2}, \cdots, e_{n}\right\}$ be an orthonormal basis for $\mathbb{Z}^{n}$. Then the characteristic vectors are those of the form $\sum_{j=1}^{n} \lambda_{j} e_{j}$ with all the $\lambda_{j}$ odd. In particular, the shortest characteristic vectors are the vectors of the form $\sum_{j=1}^{n} \lambda_{j} e_{j}$ with each $\lambda_{j} \in\{ \pm 1\}$; there are $2^{n}$ such vectors.

Now we shall find an upper bound on the number of characteristic vectors of norm $s+8$. This bound must be at least $n 2^{n}$, for the lattice $\mathbb{Z}^{n}$ has $n 2^{n}$ such vectors. (These are the vectors $\sum_{j=1}^{n} \lambda_{j} e_{j}$ with one $\lambda_{j}= \pm 3$ and all other $\lambda_{j} \in\{ \pm 1\}$.)

Lemma 3.4. Suppose $w \in \chi_{s+8}$. Define

$$
\mathcal{C}_{w}:=\left\{v \in \chi_{s+8}: w-v \in 4 L\right\} .
$$

If $n \neq 15$ then $\left|\mathcal{C}_{w}\right| \leq n$; if $n=15$ then $\left|\mathcal{C}_{w}\right| \leq 16$.

Proof. It is enough to show that $\left|\mathcal{C}_{w}\right| \leq n+1$, and then to show that equality can hold only when $n=15$.

(a) Proof of the inequality $\left|\mathcal{C}_{w}\right| \leq n+1$.

Write

$$
\begin{aligned}
w & =x_{1}+2 l_{1} \\
w & =x_{2}+2 l_{2} \\
& \vdots \\
w & =x_{m+1}+2 l_{m+1}
\end{aligned}
$$

in as many different ways as possible with $x_{i} \in \chi$ and $B\left(x_{i}, l_{i}\right)=0$ for each $i$. The list is finite because $q$ is positive definite.

Claim: $\left|\mathcal{C}_{w}\right|=m+1$. Given $v \in \mathcal{C}_{w}$, let $x=\frac{v+w}{2}$ and $l=\frac{w-v}{4}$. (So $w=x+2 l$ and $v=x-2 l$.) Then

$$
x=w+\frac{v-w}{2} \in w+2 L=\chi .
$$

But the equality $q(v)=q(w)$ then yields $q(x-2 l)=q(x+2 l)$, from which $B(x, l)=0$. This gives an injective map from $\mathcal{C}_{w}$ to rows of the list (1). Thus $\left|\mathcal{C}_{w}\right| \leq m+1$.

On the other hand, if $w=x_{i}+2 l_{i}$, then we assert that $x_{i}-2 l_{i} \in \mathcal{C}_{w}$; this vector is characteristic and in the same coset of $L / 4 L$ as $w$, and $q(w)=q\left(x_{i}-2 l_{i}\right)$. If $x_{i}-2 l_{i}=x_{j}-2 l_{j}$ then $w-4 l_{i}=w-4 l_{j}$ and so each expression for $w$ yields a different element of $\mathcal{C}_{w}$. Thus $\left|\mathcal{C}_{w}\right|=m+1$ as claimed. 
Having established this claim, to prove part (a) we need only show that $m \leq n$. One of our expressions for $w$ in (1) will be $w+0$. So without loss of generality, suppose $l_{m+1}=0$. The proof will proceed by showing $l_{1}, \cdots, l_{m}$ are linearly independent.

For $1 \leq i \leq m$ we have $q\left(x_{i}\right)+4 q\left(l_{i}\right)=s+8$. Since $x_{i}$ is characteristic, it follows that $q\left(l_{i}\right)=2$ and $q\left(x_{i}\right)=s$. Suppose $1 \leq i<j \leq m$. Because $x_{i}-2 l_{j} \in \chi$ we know $q\left(x_{i}-2 l_{j}\right) \geq s$. Hence, because $q\left(x_{i}\right)=s$, we have

$$
B\left(x_{i}, l_{j}\right) \leq q\left(l_{j}\right)=2 .
$$

We also know $l_{i} \neq l_{j}$, since the expressions in (1) are different. So $q\left(l_{i}-l_{j}\right)>0$ and therefore $B\left(l_{i}, l_{j}\right) \leq 1$. But

$$
B\left(x_{i}, l_{j}\right)+2 B\left(l_{i}, l_{j}\right)=B\left(w, l_{j}\right)=B\left(x_{j}+2 l_{j}, l_{j}\right)=4 .
$$

Thus $B\left(x_{i}, l_{j}\right)=2$ and $B\left(l_{i}, l_{j}\right)=1$ whenever $1 \leq i<j \leq m$.

We are now ready to prove that $l_{1}, l_{2}, \cdots, l_{m}$ are linearly independent. For suppose

$$
\sum_{i=1}^{m} \mu_{i} l_{i}=0
$$

with $\mu_{1} \cdots \mu_{m} \in \mathbb{Q}$. Then for each $k \leq m$ we have $B\left(\sum_{i=1}^{m} \mu_{i} l_{i}, l_{k}\right)=0$, and hence

$$
A_{m}\left(\begin{array}{c}
\mu_{1} \\
\mu_{2} \\
\vdots \\
\mu_{m}
\end{array}\right)=0
$$

where $A_{m}$ is the $m \times m$ matrix

$$
\left(\begin{array}{cccc}
2 & 1 & \ldots & 1 \\
1 & 2 & \ddots & \vdots \\
\vdots & \ddots & \ddots & 1 \\
1 & \ldots & 1 & 2
\end{array}\right)
$$

But $\operatorname{det} A_{m}=m+1$, and hence $A_{m}$ is invertible over $\mathbb{Q}$. Therefore $\mu_{1}=\mu_{2}=$ $\cdots=\mu_{m}=0$, which proves the claim.

Therefore $m \leq \operatorname{dim} \mathbb{Q} L=n$ and so $\left|\mathcal{C}_{w}\right| \leq n+1$ as required.

(b) Suppose $\left|\mathcal{C}_{w}\right|=n+1$; we will show that $n=15$.

As in the proof of part (a), write $w=x_{i}+2 l_{i}$ for each $1 \leq i \leq n$, with the $x_{i}$ distinct elements of $\chi_{s}$, and $B\left(x_{i}, l_{i}\right)=0$ for each $i$. Then the set $\left\{l_{1}, l_{2}, \cdots, l_{n}\right\}$ is a basis for $\mathbb{Q} L$, and $q\left(l_{i}\right)=2$ for each $i$. 
Write $x_{1}=\sum_{i=1}^{n} \nu_{i} l_{i}$ with $\nu_{i} \in \mathbb{Q}$. Recall that $B\left(x_{1}, l_{1}\right)=0$ and $B\left(x_{1}, l_{i}\right)=2$ for $2 \leq i \leq n$. Thus

$$
A_{n}\left(\begin{array}{c}
\nu_{1} \\
\nu_{2} \\
\vdots \\
\nu_{n}
\end{array}\right)=\left(\begin{array}{c}
0 \\
2 \\
\vdots \\
2
\end{array}\right)
$$

Solving this for $\nu_{1}, \cdots, \nu_{n}$ yields $\nu_{1}=-2\left(\frac{n-1}{n+1}\right)$ and $\nu_{2}=\cdots=\nu_{n}=\frac{4}{n+1}$ and hence

$$
x_{1}=\frac{2}{n+1}\left[-(n-1) l_{1}+2\left(l_{2}+\cdots+l_{n}\right)\right]
$$

from which we find

$$
q\left(x_{1}\right)=8\left(\frac{n-1}{n+1}\right) \in \mathbb{Z} .
$$

Since $(n-1, n+1) \leq 2$, it follows that $(n+1) \mid 16$. So $n \in\{1,3,7,15\}$. But $x_{1}$ was characteristic, so $q\left(x_{1}\right) \equiv n(\bmod 8)$. This happens only for $n=15$.

Corollary 3.5. Let $L$ be a positive definite unimodular $\mathbb{Z}$-lattice of rank $n$. If $n \neq 15$ then $L$ has at most $n 2^{n}$ characteristic elements of length $s+8$. If $L$ has rank 15 then there are at most $2^{19}$ such elements.

Proof. Regardless of the rank of $L$, the elements of $\chi$ form a coset of $L / 2 L$. Therefore $\chi$ consists of precisely $2^{n}$ cosets of $L / 4 L$. Pick an element $w_{k}$ of norm $s+8$ from each coset of $L / 4 L$ that contains such an element. Then

$$
\chi_{s+8}=\bigcup_{k} \mathcal{C}_{w_{k}} .
$$

If $n \neq 15$, Lemma 3.4 tells us there are no more than $n$ elements in each $C_{w_{k}}$. Thus there can be no more than $n 2^{n}$ elements of $\chi_{s+8}$.

If $n=15$, Lemma 3.4 tells us there are no more than 16 elements of $\chi_{s+8}$ in each $C_{w_{k}}$. Thus there can be no more than $16 \cdot 2^{15}=2^{19}$ elements of $\chi_{s+8}$.

Remark. In fact if $n=15$, calculations involving theta series show that there are at most $15 \times 2^{15}$ characteristic elements of length $s+8$.

\section{The main result}

In the first part this section, we largely follow the notation of [E2]. Let $H$ be the complex upper half plane: the set of complex numbers with strictly positive imaginary part. Define the theta series of the lattice $L$ to be

$$
\theta_{L}(t):=\sum_{v \in L} e^{\pi i q(v) t}
$$

for any $t \in H$. Then

$$
\theta_{L}(t)=\sum_{k=0}^{\infty} N_{k} e^{\pi i k t}
$$


where $N_{k}$ is the number of times $L$ represents $k$. Now let $w$ be any characteristic vector of $L$ and define

$$
\theta_{L}^{\prime}(t):=\sum_{v \in L+\frac{w}{2}} e^{\pi i q(v) t}=\sum_{k=0}^{\infty} N_{k}^{\prime} e^{\pi i k t / 4}
$$

where $N_{k}^{\prime}$ is the number of characteristic vectors of norm $k$. In [E1], Elkies relates these series by the identity

$$
\theta_{L}\left(\frac{-1}{t}+1\right)=\left(\frac{t}{i}\right)^{n / 2} \theta_{L}^{\prime}(t)
$$

The $n / 2$ power refers to the $n$th power of the principal square root.

Hecke has proved that if $L$ is a unimodular $\mathbb{Z}$-lattice, then $\theta_{L}$ is a modular form of weight $\frac{n}{2}$ and can be expressed as a weighted-homogeneous polynomial $P_{L}\left(\theta_{\mathbb{Z}}, \theta_{E_{8}}\right)$ in the modular forms $\theta_{\mathbb{Z}}$ and $\theta_{E_{8}}$ of weight $\frac{1}{2}$ and 4 repectively (see Theorem 7 , Chapter 7 of [CS] and the remark that follows it). Here, $\theta_{\mathbb{Z}}$ and $\theta_{E_{8}}$ are the theta series of the lattices $\mathbb{Z}$ and $E_{8}$. Specifically

$$
\theta_{\mathbb{Z}}=1+2\left(e^{\pi i t}+e^{4 \pi i t}+e^{9 \pi i t}+\cdots\right)
$$

and

$$
\theta_{E_{8}}=1+240 \sum_{k=0}^{\infty} \frac{k^{3} e^{2 \pi i k t}}{1-e^{2 \pi i k t}}=1+240 e^{2 \pi i t}+2160 e^{4 \pi i t}+\cdots
$$

We can express

$$
P_{L}(X, Y)=\sum_{k=0}^{l} \lambda_{k} X^{n-8 k} Y^{k}
$$

with $\lambda_{i} \in \mathbb{R}, l \leq\left[\frac{n}{8}\right]$ and $\lambda_{l} \neq 0$ and so we may write

$$
\theta_{L}(t)=\sum_{k=0}^{l} \lambda_{k} \theta_{\mathbb{Z}}^{n-8 k}(t) \theta_{E_{8}}^{k}(t)
$$

with $\lambda_{i} \in \mathbb{R}, l \leq\left[\frac{n}{8}\right]$ and $\lambda_{l} \neq 0$. Combining this with equation (2), we have

$$
\begin{aligned}
\theta_{L}^{\prime}(t) & =\left(\frac{i}{t}\right)^{n / 2} \theta_{L}\left(-\frac{1}{t}+1\right) \\
& =\sum_{k=0}^{l} \lambda_{k}\left[\left(\frac{i}{t}\right)^{(n-8 k) / 2} \theta_{\mathbb{Z}}^{n-8 k}\left(-\frac{1}{t}+1\right)\right]\left[\left(\frac{i}{t}\right)^{4 k} \theta_{E_{8}}^{k}\left(-\frac{1}{t}+1\right)\right] \\
& =\sum_{k=0}^{l} \lambda_{k} \theta_{\mathbb{Z}}^{\prime n-8 k}(t) \theta_{E_{8}}^{\prime}{ }^{k}(t) \\
& =P_{L}\left(\theta_{\mathbb{Z}}^{\prime}, \theta_{E_{8}}^{\prime}\right)
\end{aligned}
$$


But $E_{8}$ is an even lattice, hence 0 is one of its characteristic vectors. Thus $\theta_{E_{8}}=\theta_{E_{8}}^{\prime}$. So we have

$$
\theta_{L}^{\prime}=P_{L}\left(\theta_{\mathbb{Z}}^{\prime}, \theta_{E_{8}}\right)
$$

Because the characteristic vectors of $\mathbb{Z}$ (viewed as a lattice of rank one) are the odd integers, we have

$$
\theta_{\mathbb{Z}}^{\prime}=2\left(e^{\pi i t / 4}+e^{9 \pi i t / 4}+\cdots\right) .
$$

Expanding the polynomial in equation (4) now gives

$$
\theta_{L}^{\prime}(t)=\lambda_{l} 2^{n-8 l} e^{(n-8 l) \pi i t / 4}+\left(2^{8} \lambda_{l-1}+(n+232 l) \lambda_{l}\right) 2^{n-8 l} e^{(n-8 l+8) \pi i t / 4}+\cdots,
$$

where $\lambda_{l}$ and $\lambda_{l-1}$ are as in equation (3). Since $\theta_{L}^{\prime}$ encodes the number of characteristic vectors of each norm, we can deduce that if $\theta_{L}$ is expressed as in equation (3) then

$$
\left\{\begin{array}{l}
s=n-8 l \\
\left|\chi_{s}\right|=\lambda_{l} 2^{n-8 l} \\
\left|\chi_{s+8}\right|=\left(2^{8} \lambda_{l-1}+(n+232 l) \lambda_{l}\right) 2^{n-8 l} .
\end{array}\right.
$$

Theorem 4.1. Let $L$ be a positive definite unimodular $\mathbb{Z}$-lattice. Then its theta series $\theta_{L}(t)$ is a modular form of weight $\frac{n}{2}$ and can be expressed as a weightedhomogeneous polynomial $P_{L}\left(\theta_{\mathbb{Z}}, \theta_{E_{8}}\right)$ in the modular forms $\theta_{\mathbb{Z}}$ and $\theta_{E_{8}}$ of weight $\frac{1}{2}$ and 4 respectively. Here $\theta_{\mathbb{Z}}$ and $\theta_{E_{8}}$ are the theta series of the lattices $\mathbb{Z}$ and $E_{8}$. Further, if we write

$$
P_{L}(X, Y)=\sum_{k=0}^{l} \lambda_{k} X^{n-8 k} Y^{k}
$$

then $\lambda_{l} \leq 2^{8 l}$.

Proof. In light of Hecke's theorem, the only new information here is the bound on $\lambda_{l}$. Express $P_{L}(X, Y)$ as in equation (6). Then there are $\lambda_{l} 2^{n-8 l}$ shortest characteristic vectors. But Corollary 3.3 states that there are at most $2^{n}$ such vectors. Thus $\lambda_{l} \leq 2^{8 l}$.

Lemma 4.2. Let $L$ be an $n$-dimensional positive definite unimodular $\mathbb{Z}$-lattice that does not represent 1 . Suppose further that the shortest characteristic vectors of $L$ have norm $n-16$. Then

$$
\left|\chi_{s}\right|=2^{n-24}\left(2 n^{2}-46 n+N_{2}\right)
$$

(Recall that $N_{2}$ is the number of times $L$ represents 2.) 
Proof. The shortest characteristic vectors of $L$ have norm $n-16$; thus

$$
\begin{aligned}
\theta_{L}(t) & =\lambda_{0} \theta_{\mathbb{Z}}^{n}(t)+\lambda_{1} \theta_{\mathbb{Z}}^{n-8}(t) \theta_{E_{8}}(t)+\lambda_{2} \theta_{\mathbb{Z}}^{n-16}(t) \theta_{E_{8}}^{2}(t) \\
& =\lambda_{0} \theta_{\mathbb{Z}^{n}}(t)+\lambda_{1} \theta_{\mathbb{Z}^{n-8} \perp E_{8}}(t)+\lambda_{2} \theta_{\mathbb{Z}^{n-16} \perp E_{8} \perp E_{8}}(t) .
\end{aligned}
$$

We know how many times each of the numbers 0,1 and 2 are represented by the lattices $\mathbb{Z}^{n}, \mathbb{Z}^{n-8} \perp E_{8}$ and $\mathbb{Z}^{n-16} \perp E_{8} \perp E_{8}$.

So we have that

$$
\begin{aligned}
\theta_{L}(t) & =1+0 e^{\pi i t}+N_{2} e^{2 \pi i t}+\cdots \\
& =\lambda_{0}\left(1+2\left(\begin{array}{c}
n \\
1
\end{array}\right) e^{\pi i t}+2^{2}\left(\begin{array}{l}
n \\
2
\end{array}\right) e^{2 \pi i t}+\cdots\right) \\
& +\lambda_{1}\left(1+2\left(\begin{array}{c}
n-8 \\
1
\end{array}\right) e^{\pi i t}+\left(2^{2}\left(\begin{array}{c}
n-8 \\
2
\end{array}\right)+240\right) e^{2 \pi i t}+\cdots\right) \\
& +\lambda_{2}\left(1+2\left(\begin{array}{c}
n-16 \\
1
\end{array}\right) e^{\pi i t}+\left(2^{2}\left(\begin{array}{c}
n-16 \\
2
\end{array}\right)+480\right) e^{2 \pi i t}+\cdots\right) .
\end{aligned}
$$

This yields the simultaneous equations

$$
\begin{aligned}
\lambda_{0}+\lambda_{1}+\lambda_{2} & =1 \\
2 n \lambda_{0}+2(n-8) \lambda_{1}+2(n-16) & \lambda_{2} \\
= & 0 \\
2 n(n-1) \lambda_{0}+(2(n-8)(n-9)+240) \lambda_{1}+(2(n-16)(n-17)+480) \lambda_{2} & =N_{2} .
\end{aligned}
$$

Upon solving these equations, we find

$$
\lambda_{2}=\frac{2 n^{2}-46 n+N_{2}}{256} .
$$

The observations (5) now tell us

$$
\left|\chi_{s}\right|=2^{n-24}\left(2 n^{2}-46 n+N_{2}\right)
$$

as claimed.

Theorem 4.3. Let $L$ be a positive definite unimodular $\mathbb{Z}$-lattice of rank $n$. Suppose further that the shortest characteristic vectors of $L$ have norm $n-16$. Then $L=L_{0} \perp \mathbb{Z}^{r}$ for some sublattice $L_{0}$ of rank $\leq 2907$.

Proof. We may assume $L$ does not represent 1 and prove that $n \leq 2907$. By Corollary 3.3 , we know there are at most $2^{n}$ shortest characteristic vectors. But Lemma 4.2 tells us $L$ has exactly $2^{n-24}\left(2 n^{2}-46 n+N_{2}\right)$ shortest characteristic vectors. So

$$
2^{n-24}\left(2 n^{2}-46 n+N_{2}\right) \leq 2^{n}
$$

Hence

$$
2 n^{2}-46 n+N_{2} \leq 2^{24}
$$

But $N_{2} \geq 0$, hence $2 n^{2}-46 n \leq 2^{24}$ and so the integer $n$ cannot exceed 2907 . 
Lemma 4.4. Let $L$ be an $n$-dimensional positive definite unimodular $\mathbb{Z}$-lattice that does not represent 1 , and assume that the shortest characteristic vectors of $L$ have norm $n-24$. Then

$$
\left|\chi_{n-16}\right|=\left(2 n^{2}-46 n+N_{2}\right) 2^{n-24}+(n-72)\left|\chi_{n-24}\right| .
$$

Proof. Since the shortest characteristic vectors of $L$ have norm $n-24$, we may write

$$
\theta_{L}(t)=\lambda_{0} \theta_{\mathbb{Z}}^{n}(t)+\lambda_{1} \theta_{\mathbb{Z}}^{n-8}(t) \theta_{E_{8}}(t)+\lambda_{2} \theta_{\mathbb{Z}}^{n-16}(t) \theta_{E_{8}}^{2}(t)+\lambda_{3} \theta_{\mathbb{Z}}^{n-24}(t) \theta_{E_{8}}^{3}(t) .
$$

Forming three simultaneous equations exactly as in the proof of Lemma 3.1, we discover

$$
\begin{gathered}
\lambda_{2}=\frac{3 N_{3}+160 N_{2}-5568 n-6 N_{2} n+308 n^{2}-4 n^{3}}{2^{12}} \\
\lambda_{3}=\frac{-3 N_{3}-144 N_{2}+4832 n+6 N_{2} n-276 n^{2}+4 n^{3}}{3 \times 2^{12}} .
\end{gathered}
$$

Therefore

$$
\lambda_{2}=-3 \lambda_{3}+\frac{2 n^{2}-46 n+N_{2}}{2^{8}}
$$

and from the observations (5), we can express the number of characteristic vectors of length $n-16$ in terms of the number of shortest characteristic vectors:

$$
\begin{aligned}
\left|\chi_{n-16}\right| & =\left(2^{8} \lambda_{2}+(n+696) \lambda_{3}\right) 2^{n-24} \\
& =\left(2 n^{2}-46 n+N_{2}\right) 2^{n-24}+(n-72)\left(\lambda_{3} 2^{n-24}\right) \\
& =\left(2 n^{2}-46 n+N_{2}\right) 2^{n-24}+(n-72)\left|\chi_{n-24}\right|
\end{aligned}
$$

as claimed.

Theorem 4.5. Let $L$ be a positive definite unimodular $\mathbb{Z}$-lattice of rank $n$. Suppose further that the shortest characteristic vectors of $L$ have norm $n-24$. Then $L=L_{0} \perp \mathbb{Z}^{r}$ for some sublattice $L_{0}$ of rank $\leq 8388630$.

Proof. We may assume $L$ does not represent 1 and prove that the rank of $L$ is at most 8388630 .

The hypotheses imply $n \neq 15$. So Corollary 3.5 (b) tells us there can be no more than $n 2^{n}$ second shortest characteristic vectors. So by Lemma 4.4 ,

$$
\left(2 n^{2}-46 n+N_{2}\right) 2^{n-24}+(n-72)\left|\chi_{n-24}\right| \leq n 2^{n} .
$$

We may assume that $n \geq 72$ and we know that the number of shortest characteristic vectors is positive. So

$$
\left(2 n^{2}-46 n+N_{2}\right) 2^{n-24}<n 2^{n} .
$$

Rearranging,

$$
2 n^{2}-\left(46+2^{24}\right) n+N_{2}<0 .
$$


Next notice that $N_{2} \geq 0$. So inequality (8) implies $n$ can be no larger than 8388630 .

\section{Remarks}

I do not claim to have found the best possible bounds for $\mathcal{N}_{2}$ or $\mathcal{N}_{3}$. However, if $\mathcal{N}_{k}$ exists, we can see $\mathcal{N}_{k} \geq 23 k$ as follows. Consider the lattice

$$
L_{k}:=\perp_{i=1}^{k} O_{23}
$$

whose components are all copies of the 23-dimensional shorter Leech lattice $O_{23}$ (see, for example, [CS], 179). In [E2], Elkies notes that $O_{23}$ has shortest characteristic vectors of norm 15 . From this it follows that $L_{k}$ is a $23 k$-dimensional lattice with shortest characteristic vectors of norm $23 k-8 k$.

It appears that my method of bounding the number of short characteristic vectors does not yield $\mathcal{N}_{k}$ for $k \geq 4$. So Elkies' question remains open for $k \geq 4$.

Finally, by Construction A of ([CS], 137), we notice that if $k \leq 3$, there is an $n_{k}$ such that every binary self-dual code whose shadow has minimal norm $\geq \frac{(n-8 k)}{2}$ is of the form $C_{0} \oplus z^{r}$ for some code $C_{0}$ of length at most $n_{k}$.

\section{Acknowledgement}

I would like to thank my Ph.D. adviser, Larry Gerstein, for his continuing guidance and support.

\section{References}

[B] F. van der Blij, An invariant of quadratic forms mod 8, Indag. Math. 21 (1959), 291293.

[CS] J. H. Conway and N. J. A. Sloane, Sphere packings, lattices and groups, Springer, New York (1988).

[E1] N. D. Elkies, A characterization of the $\mathbb{Z}^{n}$ lattice, Math Res. Lett. 2 (1995), 321-326.

[E2] _ Lattices and codes with long shadows, Math Res. Lett. 2 (1995), 643-651.

[O'M] O. T. O'Meara, An introduction to quadratic forms, Springer, New York (1973).

[S] J.-P. Serre, A course in arithmetic, Springer, New York (1973).

Dept. of Math., University of California, SANta Barbara, CA 93106, USA

E-mail address: gaulter@math.ucsb.edu 